\title{
7 Die Unterstützung der Verantwortungsträger bei der Wahrnehmung von Verantwortung
}

\subsection{Sollen, Wollen und Können als Voraussetzung für das Wahrnehmen von Verantwortung}

Die Geschäftsführung muss drei Funktionen erfüllen, damit das Management der sozialen Verantwortung dauerhaft und erfolgreich in allen Bereichen des Krankenhauses praktiziert werden kann (Göbel 2006, S. 187f.):

1. Sie muss allen Führungskräften und Mitarbeitern mitteilen, was sie von diesen erwartet.

2. Die Mitglieder der Organisation Krankenhaus müssen davon überzeugt werden, dass sie das, was von ihnen erwartet wird, auch realisieren wollen.

3. Die Mitglieder der Organisation Krankenhaus müssen nicht nur wollen, sie müssen auch fähig sein oder dazu befähigt werden, entsprechend den Erwartungen der Geschäftsführung zu handeln.

Die Maßnahmen, mit deren Hilfe die genannten Funktionen realisiert werden können, sind Gegenstand dieses Buch-Abschnitts. Sie sind im Überblick in der Tabelle 16 dargestellt - mit Hinweis auf das Kapitel, in dem die Maßnahmen im Detail erörtert werden.

\subsection{Unterstützung des Sollens}

\subsubsection{Einführung}

Von den Wirkungen eines Unternehmensleitbildes
Das Unternehmensleitbild ist eine Selbstver- pflichtung des Krankenhauses und aller Verant- wortungsträger in diesem. Mit ihm legen sich diese auf das Einhalten bestimmter Werte und auf ein bestimmtes Verhalten gegenüber ihren Stakeholdern fest.

Die Wirkung eines Unternehmensleitbildes als Selbstverpflichtung im Sinne des Managements der sozialen Verantwortung und auf den Grad der Realisierung der Unternehmensziele ist in der Praxis und in der Literatur umstritten (Gabele; Kretschmer, S. 13ff.; Sobhani, S. 48ff.). Auffällig ist, dass sich die wissenschaftliche Auseinandersetzung mit diesem Thema in Grenzen hält, sodass auch nicht so recht deutlich wird, womit die ausgeprägten Bedenken der Gegner von Unternehmensleitbildern begründet werden.

Die Akzeptanz eines Unternehmensleitbildes ist immer dann unbefriedigend, wenn dessen Entwicklung und Implementierung nicht von der 
Tab. 16 Ziele und Maßnahmen für die Realisierung des Managements der sozialen Verantwortung

Ziele Maßnahmen

Die Führungskräfte und Mitarbeiter müssen wissen, was unter sozial verantwortlichem Handeln verstanden Diese Zielvorstellungen und damit die aus den Grundideen des Managewird und welche Handlungen erlaubt/erwünscht bzw. verboten/unerwünscht sind.

ments der sozialen Verantwortung abgeleiteten Normen werden im Regelfall zum Gegenstand eines Unternehmensleitbildes ${ }^{1}$.
Die Mitglieder der Organisation Krankenhaus müssen davon überzeugt sein, dass es für das Krankenhaus, für die in ihm aktiven Verantwortungsträger und für die Stakeholder des Krankenhauses gleichermaßen von Nutzen ist, wenn sie die Normen des Unternehmensleitbildes bei allen ihren Entscheidungen und Handlungen anwenden.
Dieses Ziel kann mit Hilfe von vier Maßnahmen erreicht werden:

- Die Überzeugungsarbeit beginnt mit der Gestaltung des Prozesses, der die Formulierung und die Implementierung des Unternehmensleitbildes zum Gegenstand hat.

- Bei der Besetzung vakanter Stellen wird darauf geachtet, dass nur noch Menschen engagiert werden, die glaubhaft machen können, dass sie sich mit den Inhalten des Unternehmensleitbildes und damit mit den Grundideen des Managements der sozialen Verantwortung identifizieren und diese beachten werden (Personalbeschaffung).

- Wenn Verantwortungsträger des Krankenhauses das Konzept des Managements der sozialen Verantwortung nicht akzeptieren (= Wollen-Defizit), wird die notwendige Überzeugungsarbeit mittels des Einsatzes spezifischer Instrumente der Personalentwicklung geleistet; das Ziel dieser Maßnahme ist, die Einstellung des Verantwortungsträgers den Anforderungen anzupassen (Personalentwicklung I).

- Um eventuell existierende Wollen-Defizite überwinden zu können, werden alternativ zu der Personalentwicklung I oder diese ergänzend geeignete Instrumente der Personalführung realisiert (Personalführung).

Die Mitglieder der Organisation Kran- Die Organisationsmitglieder bedürfen dazu sowohl der persönlichen kenhaus müssen sozial verantwort- Befähigung wie auch der institutionellen Unterstützung. liches Handeln realisieren können.
- Für den Fall einer Differenz zwischen den Anforderungen fachlicher, methodischer und sozialer Art, die sich aus der Erwartung, sozial verantwortlich zu handeln, ergeben, und den einschlägigen Fähigkeiten der Mitarbeiter (= Fähigkeits-Defizit) werden spezifische Maßnahmen der Personalentwicklung eingesetzt, um die Fähigkeitslücke schließen zu können (Personalentwicklung II).

- Es bedarf zum anderen der organisatorischen Unterstützung der Verantwortungsträger.

1 Anstelle des Begriffes „Unternehmensleitbild“ (synonym wird immer wieder auch der Begriff „Unternehmensgrundsätze“ verwendet) findet man in der Literatur und in der Praxis immer wieder auch den Begriff „Kodex“. In der vorliegenden Untersuchung wird der Begriff „Unternehmensleitbild“ verwendet, wenn es sich um ein Dokument handelt, das im Unternehmen und für das Unternehmen entwickelt worden ist. Der Begriff „Kodex“ wird verwendet, wenn Gegenstand dieses Dokuments Verhaltensgrundsätze sind, die außerhalb des Unternehmens entwickelt worden sind und mehr allgemeingültigen Charakter haben (Göbel 1992, S. 120). Zu den zuletzt genannten Dokumenten gehört zum Beispiel das Davoser Manifest.

Geschäftsführung initiiert und getragen wird. Die Geschäftsführung persönlich muss den Verantwortungsträgern vermitteln, dass die Orientierung allen Handelns an den Normen des Unternehmensleitbildes von zentraler Bedeutung für die Existenz sowie für den erfolgreichen Betrieb des Unternehmens und für seine Stakeholder von Nutzen ist. Sie muss dieses durch eigenes Han- deln vorleben. Ansonsten spielt ein Unternehmensleitbild im Unternehmensalltag sehr bald keine Rolle mehr.

Möglicherweise ist die Skepsis gegenüber einem Unternehmensleitbild der Art seiner Entwicklung und Implementierung geschuldet. Nicht selten werden Unternehmensberater damit beauftragt, ein Unternehmensleitbild zu er- 
arbeiten. Dieses entsteht dann am Schreibtisch des Beraters - gelegentlich durch die Übernahme von Texten aus für andere Unternehmen entwickelten Unternehmensleitbildern - und wird als fertiges Produkt - meist auf kostbarem Papier gedruckt - der Geschäftsführung überreicht. Möglicherweise ist es aber auch der tägliche Umgang mit dem Unternehmensleitbild, das bestenfalls von einigen Führungskräften und Mitarbeitern einmal gelesen worden ist und danach ignoriert wird.

Vielleicht sind es aber auch die Unsicherheiten hinsichtlich der inhaltlichen Gestaltung von Unternehmensleitbildern, die die Reserviertheit diesen gegenüber begründen. So gehen die Auffassungen, wie konkret die Aussagen des Unternehmensleitbildes sein sollen, weit auseinander. Einerseits wird eine möglichst konkrete Norm für eine bestimmte Handlung gefordert, weil dann die Gefahr gering sei, dass die Norm in Bezug auf das zu Veranlassende fehlinterpretiert wird. Zum anderen wird gegen den hohen Konkretisierungsgrad eingewandt, dass die Norm dann nur zufällig auf eine bestimmte Entscheidungssituation anwendbar sei. Eine mehr vage formulierte Norm werde dagegen gerne ignoriert mit dem Hinweis, dass sie im konkreten Einzelfall wenig hilfreich sei.

Die Akzeptanz und die Wirkung eines Unternehmensleitbildes hängen auch davon ab, wie gut dieses auf die aktuellen kulturellen Bedingungen der Leitungsarbeit abgestimmt ist (Kreikebaum, S. 285). Wenn die Leitsätze des Unternehmensleitbildes mit den gelebten Unternehmensgrundsätzen korrespondieren und diese gegebenenfalls so weiter entwickelt werden, dass sie sich nicht allzu weit von der bestehenden Praxis entfernen, kann mit einer guten Akzeptanz gerechnet werden.

Trotz der immer noch weit verbreiteten Skepsis nimmt die Zahl der Krankenhäuser zu, die ein Unternehmensleitbild eingeführt haben oder dieses zu tun beabsichtigen. Dieses hängt wohl vor allem damit zusammen, dass man sich damit die Unterstützung verspricht beim Ausgleich zwischen den Interessen des Unternehmens und den Individualinteressen diverser Stakeholder (Kreikebaum, S. 61). Es wird als eine Voraussetzung dafür gesehen, dass die Verantwortungsträger trotz unterschiedlicher Interessen und eventueller persönlicher Animositäten im Sinne der Realisierung der Unternehmensziele zusammenwirken.

Das Ziel eines Unternehmensleitbildes ist, für das Unternehmen Werte zu definieren, die die
Führungskräfte und die Mitarbeiter sowie andere Stakeholder zu teilen und zu leben gewillt sind (Sobhani, S. 53). Es sollen Regeln entwickelt werden für den äußerst komplizierten Umgang des Unternehmens mit den verschiedenen Stakeholdern, die an der Existenz und an dem Erfolg des Unternehmens interessiert sind.

Das Unternehmensleitbild enthält allerdings nicht nur Rechte, sondern auch Pflichten für die Führungskräfte und die Mitarbeiter (Kreikebaum, S. 81). Schließlich sind diese nicht nur Stakeholder mit Bedürfnissen, für deren Befriedigung mit dem Unternehmensleitbild die Crundlage geschaffen wird. Sie sind auch Verantwortungsträger und in dieser Funktion dem Krankenhaus zu bestimmten Handlungen und zur Übernahme von Verantwortung verpflichtet. Insofern ist das Unternehmensleitbild in einem multipersonal und arbeitsteilig organisierten Unternehmen ein Instrument, mit dessen Hilfe die Handlungen der vielen Verantwortungsträger auf das gemeinsame Unternehmensziel ausgerichtet werden können.

Nachstehend soll der Versuch gemacht werden zu zeigen, wie ein Unternehmensleitbild mit Erfolg entwickelt und eingeführt und wie dieses anschließend gelebt werden und als Basis für verantwortliches Handeln dienen kann. Dabei ist es für die Akzeptanz des Unternehmensleitbildes durch die Führungskräfte und die Mitarbeiter des Krankenhauses von zentraler Bedeutung, wie der Prozess der Entwicklung und Einführung dieses Dokuments gestaltet wird.

Weil auch in diesem Zusammenhang Entscheidungen getroffen werden, die von sozialer Verantwortung getragen werden sollten, kann auch dieser Prozess als Training für den Umgang mit dem Konzept des Managements der sozialen Verantwortung genutzt werden. Diese Auffassung wird durch Berichte aus der Praxis gestützt (Gabele; Kretschmer, S. 93). Letztere kommen gelegentlich zu dem Ergebnis, in dem Formulierungsprozess sei der Hauptnutzen des Unternehmensleitbildes zu sehen.

Das Unternehmensleitbild kann es nicht von der Stange geben. Es muss unter Berücksichtigung der personellen, kulturellen und strukturellen Besonderheiten eines Krankenhauses entwickelt werden. Das Beispiel in Kapitel 7.2.2, Beispiel eines Unternehmensleitbildes, darf deshalb auch nur als Anschauungsmaterial, als Hilfestellung für das Erarbeiten eines individuellen Unternehmensleitbildes verstanden werden. Eine andere Bedeutung sollte ihm nicht zugeschrieben 
Tab. 17 Anforderungen an die Gestaltung eines Unternehmensleitbildes

1 Das Unternehmensleitbild ist - was seine Entwicklung und Implementierung als auch die dauerhafte Orientierung des täglichen Handelns an ihm anbelangt - „Chefsache“.

2 Das Unternehmensleitbild ist das Ergebnis einer dialogischen Verständigung der Geschäftsführung des Krankenhauses mit allen Personen und/oder Institutionen, die von Entscheidungen, die auf der Basis dieses Leitbildes gefällt werden, eventuell betroffen sein werden.

3 Einen einheitlichen Informationsgrad über den Prozess des Unternehmensleitbildes haben alle Beteiligte und Betroffene nur dann, wenn dieser Prozess schriftlich dokumentiert wird. Dieses ist auch insofern wichtig, um Gerüchte und damit die Behinderung der Entwicklung des Unternehmensleitbildes vermeiden zu können.

4 Die Formulierungen des Unternehmensleitbildes müssen so einfach und eindeutig sein, dass diese auch ohne zusätzliche Erläuterungen verstanden werden können.

5 Unternehmensleitlinien werden in das Unternehmensleitbild nur dann aufgenommen, wenn sichergestellt ist, dass diese auch umgesetzt werden können. Die möglichst rasche Umsetzung auch schon einzelner Elemente des Unternehmensleitbildes hilft, dessen Akzeptanz zu sichern.

6 Sofern der Anlass für die Entwicklung und Implementierung eines Unternehmensleitbildes ein konkreter Konflikt war, sollte das Leitbild möglichst so formuliert werden, dass ähnliche Konflikte künftig aufgefangen werden können.

7 Sofern Leitbilder schon existieren - gemeint sind damit z.B. auch Führungsgrundsätze -, ist auf inhaltliche Konsistenz mit diesen zu achten. Dieses kann auch dazu führen, dass bestehende Leitbilder überarbeitet werden.

werden, weil sonst die Gefahr groß ist, dass der von der Anwendung des Unternehmensleitbildes erhoffte Nutzen ausbleibt.

Die bisherigen Ausführungen werden in der nachstehenden Tabelle 17 als Checkliste zusammen gefasst und ergänzt (Kreikebaum, S. 247).

\section{Mission, Vision und Unternehmensleitbild}

Die Frage nach den Inhalten des für ein Krankenhaus geeigneten Unternehmensleitbildes lässt sich erst dann beantworten, wenn für das Krankenhaus festgelegt worden ist, welchen Auftrag dieses zu erfüllen hat und auf welchen gesellschaftlichen Zweck hin dessen Existenz ausgerichtet ist (Sobhani, S. 47) (s. Abb. 45):

- Die Mission des Krankenhauses ist Gegenstand eines Versorgungsvertrages, der zwischen den Krankenkassen und den Krankenhäusern abgeschlossen wird. Er ist gegebenenfalls krankenhausintern zu detaillieren und um spezifische Arbeitsschwerpunkte für einzelne Leistungsbereiche zu ergänzen.

Mit dem Inkrafttreten des Gesundheitsmodernisierungsgesetzes zum 1. Januar 2004 ist den Krankenhäusern die Möglichkeit gegeben, mit den Krankenkassen und anderen Leistungserbringern des Gesundheitssystems Ver- träge abzuschließen, um sektorübergreifend Leistungen über die gesamte Versorgungskette hinweg - von der Vorsorge, der ambulanten über die stationäre medizinischen Versorgung bis hin zur Rehabilitation und der ambulanten und stationären Pflege - anzubieten. Dieses bedeutet für die Krankenhäuser, dass sie bei der Entwicklung ihrer Mission immer die gesamte Versorgungskette im Blick haben - unabhängig davon, welches Leistungsspektrum sie künftig abdecken wollen.

- Eine weitere Voraussetzung für die Entwicklung eines Unternehmensleitbildes ist die möglichst Hierarchieebenen, Berufsgruppen und Leistungsbereiche übergreifend erarbeitete - Vision, also ein Bild, das den Führungskräften sowie den Mitarbeitern eine realistische, glaubwürdige und attraktive Zukunft vermittelt (Eichhorn, S. 136). Wichtig dabei sind zwei Blickrichtungen: Zum einen legen die Geschäftsführung, die Führungskräfte und die Mitarbeiter gemeinsam fest, welche Rolle das Krankenhaus aus ihrer Sicht künftig auf dem Krankenhaus,,markt" spielen soll (s. Beispiel 1) und welche Ressourcen dafür bereit gestellt werden sollen. Zum anderen überlegen sich die Mitglieder der Organisation Krankenhaus, wie die für sie relevanten Stakeholder ihr Krankenhaus - also von au- 


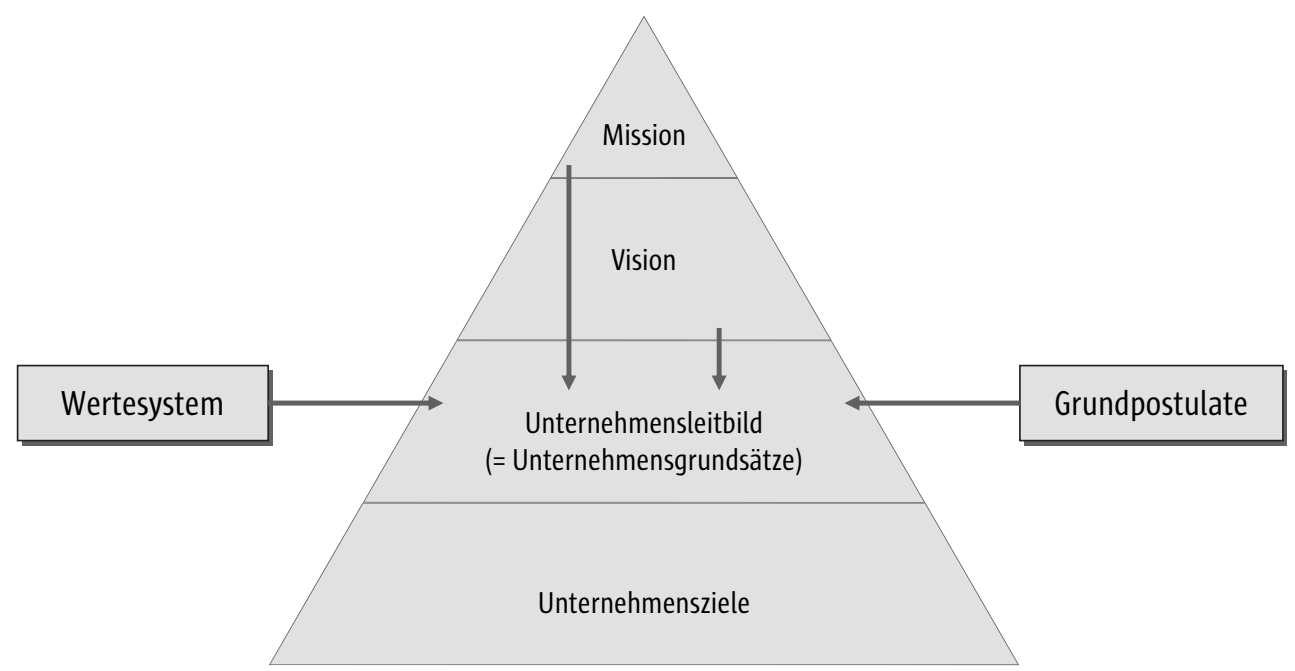

Abb. 45 Entwicklung der Unternehmensziele aus der Mission des Krankenhauses

ßen - künftig sehen sollen, was Dritte über das Krankenhaus wissen müssen (von Eiff; Stachel, S. 33) (s. Beispiel 2).

\section{Beispiel 1}

Die Krankenkassen und das Krankenhaus vereinbaren, dass das Krankenhaus Leistungen der vollständigen Versorgungskette, also Leistungen der Vorsorge, der ambulanten und der stationären medizinischen Versorgung, der Rehabilitation sowie der stationären und der ambulanten Pflege, für eine Region oder überregional für jeweils bestimmte Krankheitsarten anbietet (= Mission). Das Krankenhaus verpflichtet sich, für die Menschen in einem eindeutig umschriebenen Marktsegment medizinische Leistungen mit hoher Behandlungs- und Servicequalität und einem optimalen Preis-Leistungs-Verhältnis zu erbringen (= Element der Vision).

\section{Beispiel 2}

Das Krankenhaus möchte erreichen, auf dem Arbeitsmarkt von potenziellen Mitarbeitern als "Magnet-Hospital“ gesehen zu werden - also als Arbeitgeber, den man auch in schwierigen Zeiten anderen Arbeitsgebern bevorzugt und dem man sich gerne über eine längere Zeitspanne hinweg verpflichten will (= Element der Vision). Es entwickelt die dafür notwendigen Alleinstellungsmerkmale, die insbesondere in attraktiven Arbeitsbedingungen bestehen; zu diesen zählen nicht nur eine angemessene Entlohnung der Mitarbeiter und sozial verträgliche Arbeitszeiten, sondern vor allem auch ein zeitgemäßes Führungskonzept, (s. Naegler et al., S. 143f. und 270ff.).

- Die Steuerung des Umgangs der Führungskräfte und der Mitarbeiter untereinander als auch des Verhaltens der Institution Krankenhaus sowie der Führungskräfte und der Mitarbeiter gegenüber Patienten, Angehörigen, einweisenden Ärzten, Lieferanten und anderen Stakeholdern erfolgt mit Hilfe eines Katalogs von Werten und mit Hilfe von Grundpostulaten (s. Beispiel 3). Diese sind Vorstellungen, die von den Verantwortungsträgern z.B. Ideen und Beziehungen als wünschens- und erstrebenswert beigelegt werden. Die Werte und Grundpostulate werden mit den Aussagen über die Mission und die Vision verknüpft und in dem Unternehmensleitbild zusammengefasst.

\section{Beispiel 3}

Das Krankenhaus hat sich zu einem "Magnet-Hospital“ entwickelt (= Element der Vision); es zeichnet sich durch in mehrfacher Hinsicht äußerst attraktive Arbeitsbedingungen aus.

Nun ist eine vakante Stelle zu besetzen. Unter den vielen Bewerbern, die sich anlässlich der Stellen-Ausschreibung beworben haben, befindet sich eine größere Zahl von Kandidaten, die die Anforderungen der Stelle voll er- 
füllen und die deshalb zu einem Vorstellungsgespräch eingeladen werden. Nachdem die Entscheidung für einen der Bewerber gefällt worden war und dieser seinen Arbeitsvertrag unterschrieben hat, werden die übrigen der eingeladenen Bewerber - sofern sie dieses wünschen - darüber informiert, warum sie nicht zum Zuge kommen konnten (= Element des Unternehmensleitbildes: Grundpostulat „Begründungpflicht“).

Aus dem Unternehmensleitbild werden die Ziele des Krankenhauses entwickelt. Diese sind Aussagen über erwünschte Zustände in der Zukunft; sie sind die Grundlage für die strategische Planung und die Steuerung des Unternehmens, sie geben als gewissermaßen Leitplanken den Führungskräften und den Mitarbeitern sowie den Leistungsbereichen die Richtung, aber auch die Grenzen für deren Handeln vor. Sie sind Ausdruck der Bedeutung, die die Geschäftsleitung einzelnen Personen und/oder Institutionen zuschreibt, und können damit die Führungskräfte und die Mitarbeiter motivieren.

In dem folgenden Kapitel werden die Inhalte eines krankenhausspezifischen Unternehmensleitbildes dargestellt. Dabei kommt es darauf an zu zeigen, von welcher Art von Inhalten erwartet werden kann, dass sie im Sinne des Managements der sozialen Verantwortung nachhaltig Wirkung entfalten können. In dem diesen Themenkreis abschließenden Kapitel 7.2.3 wird beschrieben, wie der Prozess der Entwicklung eines krankenhausspezifischen Unternehmensleitbildes unter Berücksichtigung der Grundideen des Managements der sozialen Verantwortung gestaltet werden sollte. Davon hängt es nämlich ab, ob das Unternehmensleitbild ein Lippenbekenntnis bleibt oder ob es in allen Bereichen des Krankenhauses von allen seinen Mitgliedern gelebt wird.

\subsubsection{Inhalte des Unternehmensleitbildes}

\section{Überblick}

Aus der Einführung zu Kapitel 7.2 lassen sich (als Beispiel zu verstehen) vier Kategorien von Aussagen eines Unternehmensleitbildes identifizieren (Gabele; Kretschmer, S. 43):

1. der Unternehmenszweck des Krankenhauses, seine gesellschaftliche Legitimierung und die angestrebte Stellung des Krankenhauses auf dem Gesundheitsmarkt (einschließlich des spezifischen Arbeitsmarkts),
2. das Verhältnis des Krankenhauses zu seinen Stakeholdern,

3. die Einstellung der Führungskräfte und der Mitarbeiter gegenüber den in dem Krankenhaus gelebten Führungsgrundsätzen und

4. die Einstellung der Geschäftsführung zu Risiko, Wachstum und Innovation.

\section{Der Unternehmenszweck}

Der institutionelle Sinn des Krankenhauses ist die Sicherstellung der Versorgung der Bevölkerung mit medizinischen Leistungen in einem regional oder nach Krankheitsarten definierten Einzugsgebiet. Das Krankenhaus muss danach trachten, seine Existenz langfristig zu sichern, um dem zuerst genannten Auftrag nachkommen zu können. Es muss sicherstellen, dass die für die Leistungserbringung notwendige Zahl von Führungskräften und Mitarbeitern mit der erforderlichen Qualifikation und Motivation zur Verfügung steht.

\section{Das Verhältnis des Krankenhauses zu seinen Stakeholdern}

Typische Aussagen eines Unternehmensleitbildes beziehen sich auf den Umgang unter anderem mit:

- den Patienten, deren Angehörigen und den einweisenden Ärzten,

- den Führungskräften und den Mitarbeitern des Krankenhauses (beide Gruppen werden im Zusammenhang mit dem Unternehmensleitbild im Regelfall nicht getrennt behandelt, obwohl sie gelegentlich deutlich unterschiedliche Anliegen haben),

- den Personalvertretungen, die die Interessen der Führungskräfte und der Mitarbeiter gegenüber dem Eigentümer des Krankenhauses und der Geschäftsführung vertreten,

- den Vertretern des Eigentümers und der Krankenkassen,

- den Wettbewerbern,

- den Lieferanten und

- der Öffentlichkeit. 


\section{Die Einstellung der Führungskräfte und der Mitarbeiter gegenüber den in dem Krankenhaus gelebten Führungsgrundsätzen}

Eine der zentralen Voraussetzungen für sozial verantwortliches Handeln ist die Akzeptanz zeitgemäßer Führungsgrundsätze durch die Führungskräfte und Mitarbeiter und deren Umsetzen, Führungsgrundsätze nämlich, die auf der Grundlage eines in den letzten Jahren deutlich veränderten Wertekanons entwickelt worden sind.

Der Begriff „Führung“ kann sowohl institutionell als auch personell verstanden werden. Je nachdem, welcher Begriffsinhalt zugrunde gelegt wird, kommt man zu zwei unterschiedlichen Varianten von Führungsgrundsätzen:

1. In Unternehmens-Führungsgrundsätzen - synonym werden die Begriffe „Unternehmensgrundsätze“ oder „Unternehmensleitbild“ verwendet - werden die strukturellen Voraussetzungen festgelegt, die für den Vollzug der Aufgaben eines Krankenhauses notwendig sind. Dazu gehören zum Beispiel die Bestimmung der Rolle der Führungskräfte sowie die Gestaltung des Verhältnisses zwischen den Verantwortungsträgern eines Krankenhauses und den Stakeholdern.

2. Im Zentrum von Personal-Führungsgrundsätzen steht die Gestaltung des Verhältnisses zwischen der Führungskraft und deren Mitarbeitern. Es ist die ständige, glaubwürdige, informatorische Verbindung der Mitarbeiter zu den Führungskräften und der Geschäftsführung. Es ist Anstoß und Plattform für einen offenen Dialog (von Eiff; Stachel, S. 33 f.).

Unternehmensführung ist - insbesondere in einem arbeitsintensiven Dienstleistungsunternehmen wie in einem Krankenhaus - immer auch Personalführung. Insofern sind die Übergänge von Unternehmens-Führungsgrundsätzen bzw. von einem Unternehmensleitbild zu PersonalFührungsgrundsätzen immer fließend.

In dem Unternehmensleitbild werden die Erwartungen des Krankenhauses bzw. die der Geschäftsführung an die Führungskräfte und an die Mitarbeiter zum Ausdruck gebracht. Die Erwartungen beziehen sich vor allem auf das für das Realisieren der Unternehmensziele erforderliche Verhalten der Führungskräfte und der Mitarbeiter. Elemente von Unternehmensleitbildern sind ferner Aussagen über die strukturellen und kultu- rellen Bedingungen der Führungsarbeit, also zum Beispiel Aussagen zu der Delegation von Aufgaben durch die Führungskraft an ihre Mitarbeiter.

Von zentraler Bedeutung ist es daher, dass der Prozess der Entwicklung und Implementierung des Unternehmensleitbildes und der darin enthaltenen Personal-Führungsgrundsätze so gestaltet wird, dass mit deren Akzeptanz durch die Führungskräfte und die Mitarbeiter gerechnet werden kann - also auf der Grundlage einer argumentativen, dialogischen und die Bedürfnisse der Betroffenen berücksichtigenden Verständigung zwischen der Geschäftsführung sowie den Führungskräften und Mitarbeitern. Die Forderung, die Führungskräfte und Mitarbeiter an der Entwicklung des Unternehmensleitbildes zu beteiligen, ist eines der wichtigsten Elemente der Personal-Führungsgrundsätze.

Es gibt möglicherweise einen Zusammenhang zwischen der Akzeptanz und dem Umsetzen der Personal-Führungsgrundsätze und dem aktiven Umsetzen des Unternehmensleitbildes. Wenn es in einem Unternehmen Personal-Führungsgrundsätze gibt und diese aktiv gelebt werden, dann wird auch das Unternehmensleitbild von den Führungskräften aktiv vorgelebt (von Eiff; Stachel, S. 278ff.). Umgekehrt: Wenn es Personal-Führungsgrundsätze nicht gibt oder diese von den Führungskräften ignoriert werden, dann hat auch das Unternehmensleitbild keine Chance, dauerhaft wirksam zu werden.

\section{Die Einstellung der Geschäftsführung zu Risiko, Wachstum und Innovation}

Der Anteil der Krankenhäuser in privater Trägerschaft nimmt seit einigen Jahren deutlich zu. Angesichts dieser Entwicklung ist es nicht verwunderlich, dass immer häufiger die Frage gestellt wird, ob die Gewinnerzielungsabsicht neben dem Prinzip der Daseinsvorsorge Bestandteil des Unternehmensleitbildes eines Krankenhauses sein darf. Die eine Fraktion argumentiert damit, dass es im Wettbewerb um Gesundheitsdienstleistungen ohne den Anreiz für unternehmerische Gewinne herausragende Angebote nicht geben wird. Andere befürchten, dass der Cewinnerzielungsabsicht alle anderen Ziele - insbesondere die der weiteren Steigerung der Behandlungs- und Servicequalität und der Zufriedenheit der Führungskräfte und der Mitarbeiter - untergeordnet werden. 
Dieser Diskussion kann die Schärfe genommen werden, wenn die Frage etwas anders gestellt wird: Entscheidend ist dann nicht, ob die Absicht besteht, Gewinne zu erzielen oder nicht. Es fragt sich vielmehr, wie die Gewinne zustande kommen und wie sie verwendet werden. In zunehmendem Maße bekennen sich Krankenhäuser, auch solche in frei-gemeinnütziger und öffentlich-rechtlicher Trägerschaft, dazu, Gewinne erwirtschaften zu wollen (zu müssen), um sie dann in die Weiterentwicklung der personellen, apparativen und räumlichen Ausstattung des Krankenhauses investieren zu können (siehe die Leitbilder der Paul Gerhardt Diakonie e.V. und der DRK Kliniken Berlin).

Unverkennbar ist das Bemühen vieler Krankenhäuser, ihre Leistungen den Bedürfnissen ihrer Patienten immer besser anzupassen. Neue Versorgungsformen auf der Basis der Integrierten Versorgung und neue Behandlungsmethoden werden entwickelt und eingeführt. Das Ziel der Krankenhäuser ist, ihren Anteil an dem Gesamtumsatz des Gesundheitsmarktes mittels neuer Versorgungsangebote weiter auszubauen; sie begründen dieses Ziel damit, dass nur mit einer ausreichend großen Zahl von Patienten die Nachteile einer zunehmenden Spezialisierung überwunden werden können. Dem Management gut geführter Krankenhäuser ist bewusst, welche Risiken sie damit eingehen und wie diese Risiken kalkuliert und damit beherrschbar gemacht werden können.

\section{Beispiel eines Unternehmensleitbildes}

Ausgehend von den Ausführungen in den vorangegangenen Abschnitten und in Anlehnung an eine Reihe von praktischen Beispielen (DRK Kliniken Berlin, Johnson \& Johnson $\mathrm{GmbH}$, SANA Kliniken AG, Paul Gerhardt Diakonie e.V. und Wiener Krankenanstaltenverbund) - teilweise mit wörtlichen Übernahmen - könnte das Unternehmensleitbild eines Krankenhauses etwa die in der im Folgenden ausgewiesenen Inhalte haben. Dabei sind in dieses Beispiel nur die Kernaussagen aufgenommen worden. Die als Grundlage für die Entwicklung dieses Beispiels verwendeten Unternehmensleitbilder differenzieren diese Kernaussagen jeweils in mehrere, im Regelfall sehr viel konkrete Leitsätze.

\section{Unternehmensleitbild eines Krankenhauses (Beispiel)}

\section{Für wen sind wir da?}

Allem voran steht unsere Verantwortung gegenüber allen denen, die bei uns Hilfe suchen. Wir versorgen kranke, hilfs- und pflegebedürftige Menschen und bieten innen Rat und Unterstützung an, und zwar unabhängig von ihrer religiösen, politischen und kulturellen Orientierung und unabhängig von ihrem sozialen Status.

\section{Verhältnis zu unseren Patienten, Mitarbeitern und anderen Partnern}

Wir achten und schützen die Würde des Menschen. Wir sehen kranke, hilfs- und pflegebedürftige Menschen als Partner und begegnen ihnen mit Wertschätzung. Wir schaffen eine Atmosphäre des Vertrauens und der Geborgenheit.

Wir beteiligen die Patienten an allen Entscheidungen, die Diagnostik und Therapie betreffend, und binden sie in die Behandlung und Pflege mit ein. Dabei berücksichtigen wir ihre Wünsche und Lebensumstände.

Verantwortung tragen wir auch gegenüber unseren Führungskräften und allen Mitarbeitern. Jeder von innen wird als Individuum geachtet, ihre Würde wird respektiert und ihre Verdienste werden anerkannt. Sie können auf die Sicherheit ihres Arbeitsplatzes vertrauen. Wir unterstützen unsere Mitarbeiter bei der Wahrnehmung ihrer Verantwortung gegenüber ihren Familien.

Um unsere Unternehmensziele erreichen zu können, streben wir Partnerschaften aktiv an. Die Prinzipien unserer Partnerschaften lauten: Vertrauen, Zuverlässigkeit, Fairness, Kontinuität und Erfolg für beide Seiten.

\section{Einstellung zu unserem Führungskonzept}

Führung beruht auf persönlicher und fachlicher Qualifikation. Wer Führungsaufgaben übernimmt, ist Vorbild und überzeugt seine Mitarbeiter durch Kompetenz und Verantwortungsbewusstsein.

Die Führungskräfte praktizieren und fördern einen kooperativen Führungsstil. Sie nehmen Vorbild- und Fürsorgefunktion wahr. Die Mitarbeiter bilden eine Dienstgemeinschaft, die auf der Basis gegenseitiger Achtung und einer vertrauensvollen Zusammenarbeit zur Erreichung der Unternehmensziele beiträgt.

Die Mitarbeiter sind die wichtigste Ressource unseres Unternehmens. Entsprechend ihrer Position und Qualifikation erwarten wir von ihnen Einsatz und stärken ihre Eigenverantwortung. 


\section{Einstellung der Geschäftsführung zu} Risiko, Wachstum und Innovation

Wir handeln gemeinnützig und wirtschaftlich: In unserem Unternehmen fließen erwirtschaftete Mittel in die Einrichtungen zurück. Sie dienen ausschließlich der Verbesserung der Patientenversorgung, der Sicherung des Unternehmens, dem Erhalt der Arbeitsplätze oder der Erweiterung unseres Entscheidungsspielraumes.

Unser erklärtes Ziel ist es, profitables Wachstum bei gleichzeitiger Qualität in der Medizin, der Pflege und der Organisation zu erreichen. Eine wachstumsorientierte Finanzstrategie ist die Voraussetzung dafür.

\subsubsection{Einführung des Unternehmensleitbildes}

\section{Überblick}

Zu den Elementen eines Unternehmensleitbildes zählen nicht nur dessen Inhalt, sondern vor allem auch die Instrumente, die zu dessen Entstehen beitragen, und die benötigt werden, dieses mit Leben zu füllen. Ohne sie wäre das Unternehmensleitbild ein Stück Papier, von dem die gewünschte Wirkung nicht ausgehen kann.

Die Einführung des Unternehmensleitbildes erfolgt in zwei Stufen (Gabele; Kretschmer, S. 93):

1. Formulierungsprozess und

2. Diffusions- und Umsetzungsprozess.

\section{Formulierungsprozess}

Im Zusammenhang mit der Gestaltung des Prozesses der Unternehmensleitbild-Formulierung müssen drei Fragen beantwortet werden:

1. In welchen Phasen vollzieht sich der Formulierungsprozess?

2. Welche Grundpostulate sind es, die die Gestaltung des Formulierungsprozesses leiten?

3. Welche Akteure sind in welcher Funktion an diesem Prozess beteiligt?

\section{a) Phasen des Formulierungsprozesses}

Der Formulierungsprozess vollzieht sich im Regelfall in folgenden Phasen (hier folgt ein Überblick - Näheres zu einzelnen Phasen in Abschnitt c):

1. Initiieren des Projektes durch die Geschäftsführung,

2. Einsetzen der Arbeitsgruppe „Unternehmensleitbild“ und Beauftragen eines den Prozess begleitenden externen Beraters durch die Geschäftsführung,

3. Identifizieren der Leitsätze und Erarbeiten eines Rohkonzepts durch die Arbeitsgruppe,

4. Evaluieren des Rohkonzepts durch diverse, auf die Leitsätze bezogene Unter-Arbeitsgruppen,

5. Erarbeiten der endgültigen Fassung,

6. gegebenenfalls Bestätigung durch Instanzen des Krankenhaus-Trägers und Zustimmung/ Kenntnisnahme durch die Personalvertretung und

7. Beschlussfassung des Unternehmensleitbildes durch die Geschäftsführung.

\section{b) Die den Formulierungsprozess bestimmenden Grundpostulate}

Die Entwicklung eines Unternehmensleitbildes folgt mehreren Grundpostulaten des Managements der sozialen Verantwortung:

- Zum einen wird die Formulierung auf der Grundlage einer dialogischen Verständigung mit möglichst vielen der davon Betroffenen realisiert.

Das Einbeziehen möglichst vieler Stakeholder - vor allem, was externe Stakeholder wie Patienten, Krankenkassen, Banken oder Lieferanten anbelangt - stößt in der Praxis nicht selten auf kaum überwindbare organisatorische Hürden. Deshalb sind an der Formulierung des Unternehmensleitbildes meist nur die Geschäftsführung, die Führungskräfte, die Mitarbeiter und deren Vertretungen sowie gelegentlich auch ein Vertreter der Patienten und des Eigentümers beteiligt.

- Für den Formulierungsprozess wird ein Klima geschaffen - das ist vor allem die Aufgabe der Geschäftsführung -, das die Bereitschaft der beteiligten Stakeholder fördert, ihre Positionen unvoreingenommen zu präsentieren.

- Die Geschäftsführung stellt sicher, dass für die Prüfung, welche der moralischen Prinzipien in die Entwicklung des Unternehmensleitbildes einbezogen werden sollen, ausreichend Sachverstand und Zeit durch die an der Formulierung des Unternehmensleitbildes Beteiligten zur Verfügung gestellt wird (werden kann).

- DieTeilnehmer an dem Formulierungsprozess werden verpflichtet, nur solche Positionen in die Diskussion einzubringen, die sie auch begründen können.

- Schließlich erfolgt die Formulierung des Unternehmensleitbildes in einer Weise, dass die 
dort zum Ausdruck gebrachten Positionen begründet sind. Wenn das Unternehmensleitbild dadurch zu umfangreich wird und die Gefahr entsteht, dass es deswegen nicht wahrgenommen wird, empfiehlt es sich, die Begründungen in Begleit-Dokumenten festzuhalten.

\section{c) Die an dem Formulierungsprozess beteiligten Akteure und ihre Funktionen ${ }^{58}$}

Als Mitglieder der Arbeitsgruppe „Unternehmensleitbild“, die eigens für die Formulierung des Unternehmensleitbildes durch die Geschäftsführung installiert und nach dem Abschluss der Arbeiten wieder aufgelöst wird, werden im Regelfall folgende Akteure mit den nachstehend skizzierten Aufgaben berufen (Gabele; Kretschmer, S. 96):

- Die Geschäftsführung initiiert die Entwicklung und die Einführung des Unternehmensleitbildes. Die Initiative zur Entwicklung des Unternehmensleitbildes und die Beteiligung an dem Formulierungsprozess können nicht delegiert werden (von Eiff; Stachel, S. 35); das Projekt „Unternehmensleitbild“ ist „Chefsache“.

Die Geschäftsführung beteiligt sich möglichst intensiv an den Formulierungsarbeiten. Dabei reicht es, wenn sie an der Identifizierung der Leitsätze und an der Schlussredaktion teilnimmt; die Formulierungsarbeiten im Detail werden der Arbeitsgruppe übertragen. Für diese Art der Beteiligung sprechen mehrere Gründe:

- Die Geschäftsführung motiviert - gewissermaßen in ihrer Vorbild-Rolle - die Führungskräfte und die Mitarbeiter, sich an diesem Prozess zu beteiligen. Sie signalisiert Offenheit im Sinne der Förderung der Mündigkeit der Führungskräfte und der Mitarbeiter.

- Die unternehmenspolitischen Positionen des obersten Managements werden ungefiltert in den Prozess eingeführt.

- Die Geschäftsführung erfährt direkt von den Erwartungen der Führungskräfte und der Mitarbeiter und kann diese - soweit sie

58 Eine empirische Studie von Gabele und Kretschmer zeigt, dass der Formulierungsprozess in den in die Untersuchung einbezogenen Unternehmen höchst unterschiedlich organisiert ist. Es wird vorliegend eine Art Synopse versucht. akzeptiert werden können - in den Formulierungsprozess einführen.

- Sie erfährt auf diese Weise am besten und frühzeitig von eventuellen Umsetzungshindernissen und kann diese beseitigen.

- Die Beteiligung der Führungskräfte und der Mitarbeiter durch jeweils eine begrenzte und dennoch ausreichend große Zahl von Repräsentanten an dem Entwickeln des Unternehmensleitbildes ist aus zwei Gründen erforderlich:

- Als Stakeholder sind sie gefordert, ihre Bedürfnisse geltend zu machen.

- Als Verantwortungsträger achten sie darauf, dass in das Unternehmensleitbild nur die Verpflichtungen aufgenommen werden, die sie auch bewältigen können, und dass damit ihrem Bedürfnis nach Sicherheit (im Sinne des Bewältigenkönnens der in das Unternehmensleitbild aufgenommenen Verpflichtungen) entsprochen wird.

- Ein Vertreter der Personalvertretung sollte teilnehmen, damit dieser die Erwartungen der Führungskräfte und Mitarbeiter, die an dem Prozess nicht beteiligt sind, in die Diskussion einbringen kann. Zum anderen ist die Mitarbeit der Personalvertretung insofern zweckmäßig, weil diese möglicherweise gemäß Personalvertretungsgesetz beteiligt werden muss, bevor das Unternehmensleitbild durch die Geschäftsführung in Kraft gesetzt werden kann.

- Ein Ombudsmann bringt die Bedürfnisse der Patienten und der einweisenden Ärzte ein.

- Der Eigentümer wird sich mit seinen Interessen gegebenenfalls durch die Geschäftsführung vertreten lassen.

Der Formulierungsprozess wird im Regelfall durch einen Berater begleitet. Dieser übernimmt zwei Funktionen: Zum einen ist er Moderator des Prozesses und hilft in dieser Funktion, die unterschiedlichen Interessen, die im Rahmen dieses Prozesses zum Ausdruck gebracht werden, so zum Ausgleich zu bringen, dass sich die Leitsätze durch ein möglichst hohes Maß an Konkretheit auszeichnen. Er hilft zum anderen, den ersten Entwurf des Unternehmensleitbildes zu formulieren.

Der erste Entwurf der Unternehmensleitlinien sollte - bevor er einer abschließenden Bearbeitung unterzogen wird - im Sinne der Berücksichtigung der Stakeholder-Interessen einer möglichst gro- 
ßen Zahl von nicht an der Formulierungsarbeit beteiligten Führungskräften und Mitarbeitern zur Stellungnahme vorgelegt werden. Dabei sollte darauf geachtet werden, dass alle Hierarchieebenen, Berufsgruppen und Leistungsbereiche einbezogen werden.

Das dadurch ausgelöste organisatorische Problem lässt sich auf vielfältige Art und Weise lösen. Am ehesten bieten sich Klausurtagungen an, auf denen die Geschäftsführung den ersten Entwurf des Unternehmensleitbildes präsentiert und begründet. In kleineren Unter-Arbeitsgruppen werden jeweils einzelne Kategorien von Aussagen einer kritischen Analyse unterzogen und so notwendig - korrigiert und/oder ergänzt. Der Berater fasst die Stellungnahmen in einer Überarbeitung des ersten Entwurfes zusammen.

Der Prozess wird abgeschlossen durch einen Beschluss der Geschäftsführung. Zuvor holt diese - wenn notwendig - die förmliche Zustimmung oder die Stellungnahme der Personalvertretung ein. Je nach Unternehmensstruktur und -verfassung wird als Voraussetzung für das Inkrafttreten des Unternehmensleitbildes eventuell die Bestätigung durch das jeweilige Aufsichtsorgan benötigt.

\section{Diffusions- und Umsetzungsprozess}

\section{a) Phasen des Diffusions- und Umsetzungsprozesses}

Der Diffusions- und Umsetzungsprozess wird zweckmäßigerweise in den folgenden Phasen realisiert (hier folgt ein Überblick - Näheres zu einzelnen Phasen in Abschnitt b):

1. Beteiligung der Stakeholder und der Verantwortungsträger an der Formulierung des Unternehmensleitbildes,

2. Berichte über den Fortschritt der Formulierung in diversen Medien,

3. Präsentation und Begründung des Unternehmensleitbildes durch die Geschäftsführung,

4. Einbeziehen des Unternehmensleitbildes in interne Fortbildungsveranstaltungen,

5. regelmäßige Berichterstattung der Führungskräfte und Mitarbeiter über ihre Erfahrungen mit der Umsetzung des Unternehmensleitbildes und

6. Begründung aller Handlungen mit dem jeweils relevanten Leitsatz.

\section{b) Die an dem Diffusionsprozess beteiligten Akteure und ihre Funktionen}

Die Akzeptanz des Unternehmensleitbildes durch die Führungskräfte und die Mitarbeiter und damit schließlich die Wirksamkeit der in ihr festgelegten Normen für das tägliche Handeln hängt nicht nur von den in ihm festgelegten Normen und von der Art ab, wie das Unternehmensleitbild entstanden ist, sondern vor allem auch von der Art der Implementierung. Dabei wird man nicht darauf vertrauen können, dass alleine mit dem Versenden des Unternehmensleitbildes an die verschiedenen Adressaten und/oder dessen Präsentation auf der Homepage des Unternehmens - das zeigen viele Erfahrungen aus der Praxis - dieses die notwendige Aufmerksamkeit finden wird (Gabele; Kretschmer, S. 121). Weitere Maßnahmen sind erforderlich; hier folgt eine Auswahl ${ }^{59}$ :

- Die Diffusions- und Umsetzungsphase beginnt bereits mit der Formulierungsphase. Indem Führungskräfte und Mitarbeiter, aber auch andere Akteure an der Formulierung des Unternehmensleitbildes beteiligt werden, wird bereits ein erster Beitrag für das Bekanntwerden und für die Akzeptanz geleistet. Mit der Beteiligung der von der Wirkung des Unternehmensleitbildes Betroffenen, mit der Verständigung über dessen Inhalte im Dialog mit den Betroffenen und mit der Begründung der Leitsätze in dem weiter oben empfohlenen Rückkopplungsprozess wird ein gutes Klima für die Realisierung der weiteren Diffusionsund Umsetzungsschritte geschaffen.

- Diese Wirkung kann verstärkt werden, wenn bereits von Beginn der Formulierungsphase an in der Hauszeitung, über das Intranet oder über andere Medien regelmäßig über das Projekt und dessen Fortschritt berichtet wird. Der Berichterstatter sollte ein Mitglied der Geschäftsführung sein.

Mit der zeitnahen Information wird zweierlei erreicht: Zum einen verhindert man das Entstehen von Gerüchten und dadurch ausgelöste Behinderungen des Formulierungsprozesses. Zum anderen markiert die Geschäftsführung Positionen, von denen sie im weiteren Verlauf

59 In einer empirischen Studie von Gabele und Kretschmer wird gezeigt, wie höchst unterschiedlich Unternehmen den Prozess der Diffusion und Umsetzung gestaltet haben (Gabele, Kretschmer, S. 121ff.). 
der Formulierungsphase nur noch schwer wieder abrücken kann.

- Von zentraler Bedeutung für die Akzeptanz des Unternehmensleitbildes ist dessen Präsentation durch ein Mitglied der Geschäftsführung. Dieses stellt das Unternehmensleitbild im Detail vor und begründet dieses.

- Es empfiehlt sich, das Unternehmensleitbild als Unterrichtsgegenstand in die betriebliche Fortbildung zu integrieren.

- Ein Unternehmensleitbild ist auch nach der Beschlussfassung durch die dafür zuständigen Gremien nicht immer vollständig und endgültig (von Eiff; Stachel, S. 39) und muss gegebenenfalls adaptiert werden. Es empfiehlt sich deshalb, die Führungskräfte und die Mitarbeiter regelmäßig nach deren Erfahrung mit der Umsetzung des Unternehmensleitbildes zu fragen. Auf diese Weise erhält die Geschäftsführung wertvolle Hinweise über Schwächen des Unternehmensleitbildes und auf personelle und strukturelle Unzulänglichkeiten, die das Wahrnehmen von Verantwortung behindern.

Mittels der Unternehmensleitbild-Evaluierung werden die Führungskräfte und die Mitarbeiter immer wieder an dessen Berücksichtigung im Krankenhaus-Alltag erinnert.

- Eine weitere Möglichkeit, das Unternehmensleitbild immer wieder in das Bewusstsein der Führungskräfte und der Mitarbeiter zu rücken, besteht darin, die Führungskräfte und die Mitarbeiter zu verpflichten, bei jedem Erzeugen eines Dokumentes - dabei kann es sich zum Beispiel um einen Brief an Dritte, um das Protokoll über eine Besprechung, um eine Hausmittelung u.a.m. handeln - auf diesem festzuhalten, mit welchem Leitsatz oder mit welchen Leitsätzen der Vorgang, der dokumentiert wird, im Zusammenhang steht. Diese Vorgehensweise zwingt dazu, bei jeder Handlung darüber nachzudenken, ob die Handlung durch die Normen des Unternehmensleitbildes gedeckt ist.

\subsection{Unterstützung des Wollens}

\subsection{1 Überblick}

Die Bereitschaft der Führungskräfte und der Mitarbeiter, sich mit dem Konzept des Managements der sozialen Verantwortung zu identifizieren und entsprechend den im Unternehmensleitbild festgelegten Normen handeln zu wollen, kann mit Hilfe mehrerer Maßnahmen erreicht werden (s. Tab. 16):

1. Wenn eine Stelle - aus welchen Gründen auch immer - vakant ist und besetzt werden soll, kann die Rekrutierung so gestaltet werden, dass nur Bewerber um die freie Stelle zum Zuge kommen können, deren Bereitschaft gegeben ist, sich mit den Anforderungen des Konzepts „Management der sozialen Verantwortung“ $\mathrm{zu}$ identifizieren und diese bei ihren täglichen Handlungen zu respektieren (s. Kap. 7.3.2, Personalbeschaffung).

2. Mittels geeigneter Maßnahmen der Personalentwicklung wird versucht, die Einstellung der gegenwärtigen Führungskräfte und Mitarbeiter gegenüber der Crundidee des Managements der sozialen Verantwortung - soweit dieses notwendig ist - im positiven Sinne zu verändern (s. Kap. 7.3.3, Personalentwicklung I).

3. Die Personalführung trägt durch spezifische Personalführungsleistungen dazu bei, dass die Führungskräfte und die Mitarbeiter entsprechend der Crundidee des Managements der sozialen Verantwortung handeln wollen (s. Kap. 7.3.4, Personalführung).

4. Schließlich ist es die Art der Beteiligung der Führungskräfte und der Mitarbeiter an dem Formulierungsprozess sowie an dem Diffusions- und Umsetzungsprozess, die das Wollen $\mathrm{zu}$ sozial verantwortlichem Handeln fördert (darauf wurde im Kap. 7.2.3, Formulierungsprozess, sowie Diffusions- und Umsetzungsprozess bereits hingewiesen; eine erneute Erörterung kann deshalb unterbleiben).

\subsubsection{Personalbeschaffung}

\section{Überblick}

Der Prozess der Personalbeschaffung beginnt mit der Personalsuche (Naegler et al., S. 146f.). Er wird nach der Personalauswahl mit der Personaleinstellung fortgesetzt und endet mit dem Personalbeschaffungscontrolling (s. Tab. 18); nur die beiden zuerst genannten Phasen und das Personalbeschaffungscontrolling sind Gegenstand der weiteren Betrachtung. 
Tab. 18 Phasen/Teil-Phasen der Personalbeschaffung

\begin{tabular}{|l|l|l|}
\hline Phasen der Personalbeschaffung & Teil-Phase \\
\hline Personalsuche & $\begin{array}{l}\text { Stellenbeschreibung } \\
\text { Entscheidung über die Personalsuche auf dem internen oder } \\
\text { auf dem externen Arbeitsmarkt } \\
\text { Stellenanzeige }\end{array}$ \\
\hline Personalauswahl & $\begin{array}{l}\text { Analyse der Bewerbungsunterlagen } \\
\text { Vorstellungsgespräch } \\
\text { Ergänzende Tests } \\
\text { Personalvorschlag }\end{array}$ \\
\hline (Personaleinstellung) & \begin{tabular}{l}
-.. \\
\hline Personalbeschaffungscontrolling
\end{tabular} & $\begin{array}{l}\text { sach- und zeitgerechte Besetzung einer vakanten Stelle } \\
\text { nachhaltige Eignung des neuen Mitarbeiters }\end{array}$ \\
\hline $\begin{array}{l}\text { Realisierung des Beschaffungsprozesses im Sinne des Managements } \\
\text { der sozialen Verantwortung }\end{array}$ \\
\hline
\end{tabular}

\section{Personalsuche}

Die Personalsuche beginnt mit der Formulierung der Stellenbeschreibung durch den Vorgesetzten. In der Stellenbeschreibung werden die Anforderungen - unter Berücksichtigung der sich aus dem Konzept des Managements der sozialen Verantwortung ergebenden Spezifika (s. Tab. 19) - ausgewiesen, die ein Bewerber erfüllen muss, wenn er auf die ausgeschriebene Stelle berufen werden möchte. Der Vorgesetzte gibt in der Stellenbeschreibung zusätzlich an, welche aus seiner Sicht die Schlüsselanforderungen sind, die gewissermaßen als KO-Kriterien bei der Personalauswahl verwendet werden, und mit welchem Gewicht die Anforderungen zu der Bewertung der BewerberFähigkeiten herangezogen werden sollen.

Nur wenn das Anforderungsprofil ausreichend konkret und stellenangemessen formuliert wird, besteht eine Chance für das Finden des geeigneten Bewerbers. Und nur dann ist es möglich, das Ergebnis des Auswahlprozesses - z.B. gegenüber der Personalvertretung, deren Zustimmung zu der Einstellung benötigt wird, und gegebenenfalls gegenüber den Bewerbern, die nicht zum Zuge gekommen sind - zu begründen.

Auf der Grundlage der Stellenbeschreibung kann festgestellt werden, auf welchem - dem internen und/oder dem externen - Arbeitsmarkt die benötigten Mitarbeiter wahrscheinlich rekrutiert werden können und auf welchem Arbeits- markt deshalb gesucht werden soll. Im Regelfall wird man den vakanten Arbeitsplatz auf beiden Arbeitsmärkten ausschreiben.

Beide Suchstrategien haben Vor- und Nachteile (Naegler et al., S. 146f.). Mit Blick auf das Management der sozialen Verantwortung empfiehlt es sich - wenn die Suche dort nicht von vorneherein als völlig aussichtslos angesehen werden muss - die Personalsuche vor allem auch auf dem internen Arbeitsplatz. Die Geschäftsführung signalisiert dadurch ihre Bereitschaft, einen Beitrag leisten zu wollen für die Befriedigung der Bedürfnisse der gegenwärtigen Führungskräfte und Mitarbeiter nach Sicherheit des Arbeitsplatzes, nach Zugehörigkeit (man kann im Unternehmen bleiben und dort Karriere machen), nach Wertschätzung (im Sinne von Anerkennung, dass es im Unternehmen ausreichend qualifizierte Bewerber für die vakante Position gibt) und nach Selbstverwirklichung (durch die Übernahme einer neuen Aufgabe wird die Chance zur Entfaltung der eignen Persönlichkeit eröffnet).
Mit dieser Entscheidung demonstriert die Ge- schäftsführung sozial verantwortliches Handeln und motiviert die vorhandenen Mitarbeiter durch Vorbild, es ihr durch die Berücksichtigung der Be- dürfnisse der von ihren Entscheidungen Betroffe- nen gleich zu tun. 
Tab. 19 Ausschnitt eines Anforderungsprofils - Anforderungen unter Berücksichtigung des Managements der sozialen Verantwortung

\begin{tabular}{|c|c|}
\hline Klassifikation der Anforderungen & Anforderungen an \\
\hline (Fachliche Anforderungen) & $\ldots$ \\
\hline Methodische Anforderungen & $\begin{array}{l}\text { die Fähigkeit, die Bedürfnisse und Anliegen Betroffener zu erkennen, zu bewerten } \\
\text { und bei Entscheidungen zu berücksichtigen, } \\
\text {... }\end{array}$ \\
\hline Soziale Anforderungen & $\begin{array}{l}\text { Dialogfähigkeit, } \\
\text { abweichende Meinungen und Ansichten respektieren, } \\
\text { Kooperationsvermögen, } \\
\text { Verhalten, das durch Achtung gegenüber den Bedürfnissen anderer und durch } \\
\text { Solidarität gekennzeichnet ist, } \\
\text { Toleranz, } \\
\text { Konsenswillen, Ehrlichkeit, Offenheit und wechselseitige Anerkennung (Göbel 2006, } \\
\text { S. 229), } \\
\text { Verantwortungsbewusstsein, } \\
\text { Einfühlungsvermögen, } \\
\text { Bereitschaft, an der Entwicklung des Unternehmens mitzuwirken, } \\
\text {... }\end{array}$ \\
\hline \multicolumn{2}{|l|}{ Anmerkung: } \\
\hline $\begin{array}{l}\text { Der hier festgehaltene Ausschnitt ein } \\
\text { nagements der sozialen Verantwortu } \\
\text { Der Schwerpunkt der für das Thema s } \\
\text { die fachlichen Anforderungen wird d }\end{array}$ & $\begin{array}{l}\text { derungsprofils zeigt beispielhaft die Anforderungen, die ein Stelleninhaber aus der Sicht des Ma- } \\
\text { tzlich zu den übrigen Anforderungen - fachlicher, methodischer und sozialer Art - erfüllen sollte. } \\
\text { hen Anforderungen liegt naturgemäß bei den methodischen und sozialen Anforderungen; auf } \\
\text { ier nicht eingegangen. }\end{array}$ \\
\hline
\end{tabular}

Die Stellenanzeige basiert auf der Stellenbeschreibung. Sie enthält möglichst umfassende Informationen über das Krankenhaus und über die Organisationseinheit, in der die zu besetzende Stelle verortet ist. Es werden die Ziele und die Aufgaben der Stelle beschrieben. Sie informiert über jene Anforderungen, die der Vorgesetzte als Schlüsselanforderungen gekennzeichnet hat und die bei der Bewertung der Bewerbung Verwendung finden.

\section{Personalauswahl}

Der Prozess der Personalauswahl beginnt mit der formalen und inhaltlichen Analyse der Bewerbungsunterlagen. Die inhaltliche Analyse basiert zum einen auf den Angaben der Stellenanzeige und zum anderen auf zusätzlichen Informationen der Stellenbeschreibung darüber, wie die verschiedenen Anforderungen bei der Bewertung der Bewerbung gewichtet werden sollen. Das Er- gebnis der Analyse wird dokumentiert, um es den Bewerbern gegebenenfalls erläutern und begründen zu können.

Das Ziel des dann folgenden Vorstellungsgesprächs ist es vor allem, den Bewerber für das Krankenhaus zu begeistern. Ferner gilt es herauszufinden, ob der Bewerber bereit und in der Lage ist, die Grundidee des Managements der sozialen Verantwortung zu akzeptieren und entsprechend verantwortungsvoll zu handeln. Darüber hinaus geht es darum, Informationen zu erhalten, die die Bewerbungsunterlagen ergänzen und erläutern, die Belastungsfähigkeit des Bewerbers in Stresssituationen zu erkunden und dem Bewerber $\mathrm{Ge}$ legenheit zu geben, seinen künftigen Aufgabenbereich kennen zu lernen (wenn letzteres nicht schon in Vorgesprächen bzw. im Rahmen einer Betriebsbesichtigung erfolgt ist). Von besonderem Interesse ist es, die spezifischen Bedürfnisse des Bewerbers kennen zu lernen, um diese gegebenenfalls bei der endgültigen Gestaltung der wahrzunehmenden Aufgabe und des Arbeitsvertrages 
berücksichtigen zu können (s. Grundpostulat „Berücksichtigung der Interessen Betroffener“).

Das möglichst detailliert beschriebene Anforderungsprofil ist die Grundlage für das Vorstellungsgespräch. Mit diesem versuchen sowohl der Arbeitgeber als auch der Bewerber im Sinne der argumentativen, dialogischen Verständigung herauszufinden, ob das Fähigkeitsprofil des Bewerbers mit dem Anforderungsprofil zusammengeführt werden kann und welche Entwicklungspotenziale der Bewerber gegebenenfalls mitbringt, um eine eventuell bestehende Fähigkeitslücke beseitigen zu können.

Unabhängig davon, wie das Vorstellungsgespräch im Einzelnen gestaltet wird (Einzelheiten dazu s. Naegler et al., S. 153ff.), kommt es darauf an, ein Klima zu schaffen, das ein großes Maß an Wertschätzung dem Bewerber gegenüber zum Ausdruck bringt. Es bedarf eines Klimas, in dem der Bewerber ermutigt wird, in einen Dialog mit den Vertretern des Arbeitgebers über Mission, Vision und das Unternehmensleitbild des Unternehmens und über Ziele und Aufgaben der zu besetzenden Stelle einzutreten (s. die Grundpostulate „Förderung der argumentativen, dialogischen Verständigung “ und „Förderung der Mündigkeit der Stakeholder"). Nur anhand dieses offen geführten Dialogs wird es gelingen herauszufinden, ob ein Bewerber dem Anforderungsprofil genügt.

Das Vorstellungsgespräch wird abgeschlossen mit dem Angebot, den nicht erfolgreichen Bewerbern im Anschluss an eine gewisse Wartezeit zu erläutern, warum die Auswahlentscheidung nicht zu ihren Gunsten gefällt werden konnte (s. Grundpostulat „Begründungspflicht“).

Das Vorstellungsgespräch hat nicht nur eine Analyse-, sondern auch eine therapeutische Funktion. An dem Verhalten der Vertreter des Krankenhauses lernt der Bewerber, was in diesem Krankenhaus unter sozial verantwortlichem Handeln verstanden wird, um dieses später praktizieren zu können. An den Reaktionen des Bewerbers können die Vertreter des Arbeitsgebers feststellen, ob sie damit rechnen können, dass sich der Bewerber mit den Normen des Unternehmensleitbildes identifizieren wird können und welche Maßnahmen gegebenenfalls erforderlich sind, um vorhandene Wollen- und Fähigkeits-Defizite beseitigen zu können.

Vielen Krankenhäusern ist das Vorstellungsgespräch nicht aussagefähig genug. Sie möchten deshalb anhand ergänzender Tests und insbesondere mit Hilfe eines Assessment Centers mehr über den Bewerber erfahren. Vor allem das Assessment Center erfreut sich zunehmender Beliebtheit. Durch eine Vielzahl von Studien konnte festgestellt werden konnte, dass die Validität der Prognose-Ergebnisse im Vergleich zu den Ergebnissen anderer Auswahlverfahren am höchsten ist (Holtbrügge, S. 101). Die auf dem Ergebnis eines Assessment Centers aufbauende Auswahlentscheidung dient deshalb auch der Befriedigung des Bedürfnissen des Vorgesetzten, der diese Entscheidung fällt, nach Sicherheit, nach Sicherheit nämlich nach dem erfolgreichen Bewältigen des Auswahlprozesses.

Der Prozess der Personalauswahl wird abgeschlossen mit der Entscheidung für einen der Bewerber. Diese Entscheidung und die Gründe, die zu ihr geführt haben, werden sorgfältig dokumentiert. Die Dokumentation ist die Crundlage für eventuelle Gespräche mit den nicht zum Zuge gekommenen Bewerbern, für die Begründung der Auswahlentscheidung gegenüber der Personalvertretung und eventuell anderen Gremien sowie möglicherweise vor einem Arbeitsgericht, wenn ein Bewerber geltend macht, das Ergebnis der Auswahlentscheidung sei zu seinen Ungunsten fehlerhaft.

Mit der an den Grundpostulaten des Manage-
ments der sozialen Verantwortung ausgerich-
teten Gestaltung dieses Elements der Perso-
nalbeschaffung zeigt die Geschäftsführung den
künftigen Mitarbeitern, was sie unter sozial ver-
antwortlichem Handeln versteht. Sie motiviert
diese durch dieses Vorbild zu vergleichbarem
künftigem Handeln.

\section{Personalbeschaffungscontrolling}

Der Gegenstand des Personalbeschaffungscontrollings ist zum einen der Prozess der Personalbeschaffung und zum anderen das Ergebnis des Auswahlprozesses:

- Der Controller vergewissert sich, ob vakante Stellen rechtzeitig besetzt werden. Das Personalbeschaffungscontrolling gibt Antwort auf die Frage, ob die Ausschreibung zu besetzender Stellen und die Vorstellungsgespräche im Sinne der dafür skizzierten Normen gestaltet werden.

- Zu den Gegenständen des Controllings zählt die Einstellung der ausgewählten Bewerber 
hinsichtlich der Normen des Unternehmensleitbildes und die Fähigkeiten der neuen Mitarbeiter sowohl in Bezug auf sozial verantwortliches Handeln als auch mit Blick auf die sich aus der Aufgabe ergebenden Anforderungen fachlicher, methodischer und sozialer Art.

\subsubsection{Personalentwicklung I (Einstellungsentwicklung)}

Personalentwicklung als Einstellungsentwicklung wird immer dann notwendig, wenn ein Mitarbeiter als Verantwortungsträger zwar über die Fähigkeiten - fachlicher, methodischer und sozialer Art - verfügt, die zur erfolgreichen Wahrnehmung der ihm übertragenen Aufgabe benötigt werden, die aber als sozial verantwortliches Handeln nicht zur Wirksamkeit kommen, weil die Mitarbeiter die Notwendigkeit des Einsatzes des bessern Management-Konzepts nicht akzeptieren (= Wollen-Defizit). Dieses Defizit ist Ausdruck fehlenden Leistungswillens, der durch grundlegende Wertvorstellungen der Mitarbeiter bestimmt ist - durch Wertvorstellungen, die nicht im Einklang stehen mit den Werten des Unternehmensleitbildes.

Das Ziel der Einstellungsentwicklung ist (Wittmann, S. 187), die Lernbereitschaft der Mitarbeiter zu fördern. Sie sollen dazu gebracht werden, neuen Konfliktsituationen offen und gestaltungswillig gegenüberzutreten. Es soll die Bereitschaft entwickelt werden, eigene Entscheidungen und Handlungen selbstkritisch daraufhin zu überprüfen, ob die Crundpostulate sozial verantwortlichen Handelns, insbesondere das der Berücksichtigung der relevanten Stakeholder-Anliegen, ausreichend berücksichtigt worden sind, und diese gegebenenfalls zu korrigieren.

Diese Art der Personalentwickelt verlangt nach dem Einsatz geeigneter Methoden über einen längeren Zeitraum hinweg (Holtbrügge, S. 105). Der Grundgedanke dabei muss sein - anders als bei der Entwicklung von Fähigkeiten -, die Mitarbeiter als ganze Persönlichkeiten zu fördern (Wittmann, S. 186).

Wenn die Art und der Umfang des Wollen-Defizits festgestellt und die Ziele der Personalentwicklung festgelegt worden sind, ist zu klären, ob die Mitarbeiter, für die ein Wollen-Defizit festgestellt worden ist, über ausreichendes Entwicklungspotenzial verfügen und ob es sich lohnt, in deren Weiterentwicklung zu investieren. Es be- darf schließlich der Festlegung des Budgets für die Personalentwicklung und - wenn nicht alle in Betracht kommenden Mitarbeiter in die Personalentwicklung einbezogen werden können - der Beantwortung der Frage, nach welchen Kriterien die Adressaten der Personalentwicklung ausgewählt werden sollen (Werden die Adressaten ausschließlich nach dem Entwicklungsbedarf ausgewählt oder werden bestimmte Berufsgruppen privilegiert oder dient die Personalentwicklung der Begabtenförderung? Scholz, S. 509). Unter Berücksichtigung aller dieser Einflussfaktoren werden schließlich die Adressaten der Qualifizierungsmaßnahmen und die Art und der Umfang der Entwicklungsmaßnahmen festgelegt.

Personalentwicklung als Individualentwicklung wäre allerdings zu eng gesehen, würde man sie ausschließlich mit gezielt geplanten und nach ihrer Durchführung evaluierten Qualifizierungsmaßnahmen identifizieren. Jede Maßnahme der Personalarbeit, insbesondere die der Personalführung trägt zur Weiterentwicklung der Mitarbeiter bei, hinterlässt ihre Spuren in der Belegschaft (Neuberger, S. 157) - und zwar im positiven wie im negativen Sinne. Dies bedeutet: Die Führungskräfte können durch geeignete Personalführungsleistungen einen beträchtlichen Beitrag zur Personalentwicklung leisten und damit manche kostspielige Qualifizierungsmaßnahme überflüssig machen. Dies bedeutet aber auch: Interesse wecken bei den Führungskräften für den Erwerb und den Ausbau der Führungskompetenz und deren Umsetzung in praktisches Handeln ist die effizientere Personalentwicklungs-Strategie.

\subsubsection{Personalführung}

Die Personalführung trägt durch spezifische Personalführungsleistungen, durch die direkte, persönliche Interaktion zwischen der Führungskraft und deren Mitarbeitern zu der Realisierung der dem Krankenhaus gesetzten Ziele bei, also auch $z u$ dem Realisieren des Managements der sozialen Verantwortung. Sie leistet einen Beitrag zur Überwindung der möglicherweise bestehenden Diskrepanz zwischen den Zielen des Krankenhauses und den Anliegen der Mitarbeiter. Sie erreicht damit, dass sich die Mitarbeiter mit der Crundidee des Managements der sozialen Verantwortung identifizieren und in diesem Sinne verantwortlich handeln.

Die Führungskraft nutzt dabei ihre Rolle als Vorbild. Sie überzeugt ihre Mitarbeiter von der 
Nützlichkeit des neuen Management-Konzepts, indem sie Personalführung unter Berücksichtigung der Interessen der von ihr geführten Mitarbeiter und als Ergebnis einer argumentativen, dialogischen Verständigung praktiziert und ihre Führungsentscheidungen begründet.

Die Personalführung als Interaktion zwischen einer Führungskraft und ihren Mitarbeitern ist Teil eines komplexen Systems (s. Abb. 46), dessen Elemente sorgfältig aufeinander abgestimmt sein müssen. Nur dann können die Führungsleistungen erbracht werden, die als Voraussetzung für sozial verantwortliches Handeln notwendig sind. Die Gestaltung dieser Elemente war bereits in anderen Zusammenhängen Gegenstand der vorliegenden Erörterung. Es wird deshalb auf die dortigen Ausführungen verwiesen (die Nummern der Kapitel in der folgenden Übersicht verweisen auf die jeweiligen Textstellen).

Das Führungskonzept besteht unter anderem aus

- der Führungsorganisation (s. Kap. 6.4.3, Organisation, und Abschnitt Anforderungen an die Organisation),

n dem Führungsstil (s. Kap. 6.4.3, Personalarbeit, und Abschnitt Anforderungen an das Personalmanagement) und

- der Führungstechnik(s. Kap. 6.4.3, Personalarbeit, und Abschnitt Anforderungen an das Personalmanagement).

\subsection{Unterstützung des Könnens}

\subsubsection{Personalentwicklung II (Fähigkeitsentwicklung)}

Das Management der sozialen Verantwortung evoziert, ausgehend von den Fähigkeiten, über die Mitarbeiter, von denen sozial verantwortliches Handeln erwartet wird, verfügen müssen (s. Tab. 19), spezifische Entwicklungsinhalte. Vor allem geht es darum, die Mitarbeiter diskursfähig zu machen (Wittmann, S. 187). Das bedeutet einerseits, die Mitarbeiter darin zu bestärken, Konflikte in einem multipersonal, arbeitsteilig organisierten Unternehmen als selbstverständlich zu akzeptieren, diese offen anzusprechen und sie aktiv zu lösen. Damit die genannten Akteure dazu fähig sind, müssen deren kognitive, soziale und kommunikative Fähigkeiten ausgebaut werden. Von zentraler Bedeutung sind dabei Fähigkeiten wie Dialogfähigkeit, Kooperationsvermögen, Achtung gegenüber den Bedürfnissen anderer, Toleranz, Solidarität und Konsenswillen.

Der Prozess der Personalentwicklung im Sinne der Fähigkeitsentwicklung entspricht prinzipiell dem der Einstellungsentwicklung (Näheres dazu s. Kap. 7.3.3); er bedingt einen entsprechend hohen Ressourcen-Einsatz. Auch hier muss deshalb zunächst geklärt werden, ob es sich angesichts seines spezifischen Entwicklungspotenzials lohnt, in die Entwicklung eines Mitarbeiters zu investieren.

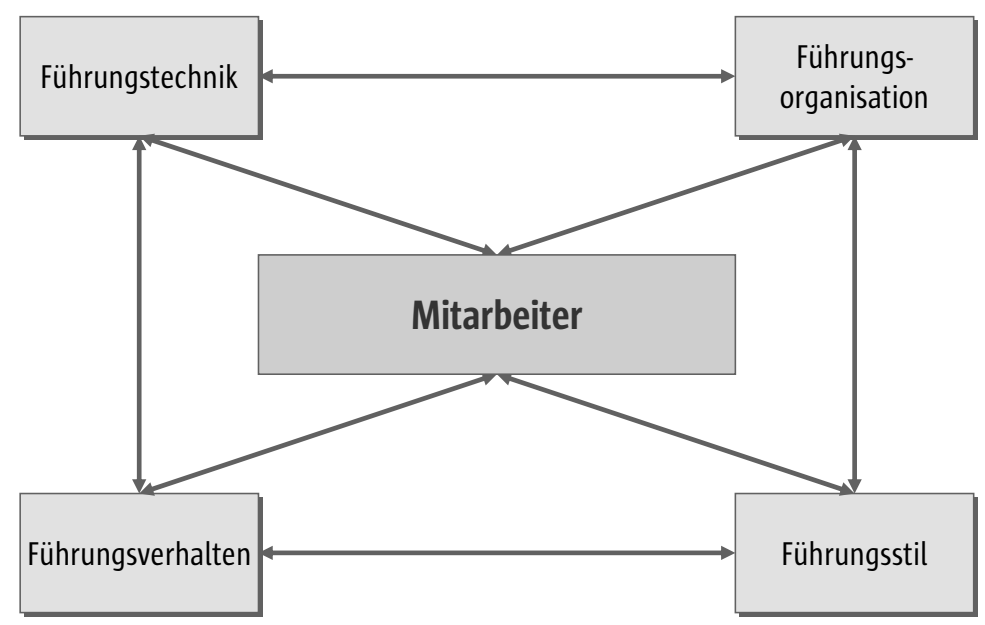

Abb. 46 Führungskonzept 
Schon die kurze Charakterisierung des Prozesses der Personalentwicklung lässt erahnen, wie viel Konfliktpotenzial dieser Prozess bereithält. Es bietet sich deshalb an, die notwendigen Entscheidungen im Sinne des Managements der sozialen Verantwortung herbeizuführen und neben den Interessen des Krankenhauses auch die Bedürfnisse der Mitarbeiter nach zum Beispiel Sicherheit, Wertschätzung und Selbstverwirklichung zu berücksichtigen. Das Mitarbeiterorientierungsgespräch ist ein geeignetes Instrument, um den Ausgleich der Interessen im Dialog zu realisieren (Naegler et al., S. 28off.).

Was die Methoden der Personalentwicklung anbelangt, wird auf die einschlägigen Ausführungen in Kapitel 7.3.3 verwiesen.

\subsubsection{Organisationale Hilfen}

\section{Einführung}

Es sind zwei Formen der aufbauorganisatorischen Grundstruktur - die Primärorganisation und die Sekundärorganisation -, derer die Führungskräfte und deren Mitarbeiter als stabile Basis bedürfen, um sozial verantwortlich handeln zu können.

Dabei wird unter Primärorganisation jene Grundstruktur einer Organisation verstanden, die es den Mitarbeitern des Krankenhauses ermöglicht, ihre Routineaufgaben zielkonform und vor allem verantwortungsbewusst wahrzunehmen. Wie die Aufbauorganisation als Primärorganisation gestaltet werden sollte, um im Sinne des Managements der sozialen Verantwortung Wirksamkeit entfalten zu können, wurde in Kapitel 6.4.3, Organisation, ausführlich behandelt.

Spezialprobleme - wie zum Beispiel die Einführung des Unternehmensleitbildes und dessen Weiterentwicklung oder die Lösung einzelner, im Zusammenhang mit dem Management der sozialen Verantwortung stehender Probleme - lassen sich im Rahmen der Primärorganisation meist nicht lösen. Dies geschieht deshalb auf der Basis einer die Primärorganisation des Unternehmens ergänzenden Sekundärorganisation (Staehle, S. 723).

Diese kann unterschiedlich ausgeprägt sein:

1. Für die Lösung eines Problems wird eine Arbeitsgruppe, die gegebenenfalls auch nur mit einer Person besetzt ist, eingesetzt. Diese wird wieder aufgelöst, wenn das Problem gelöst worden ist. Als Beispiel für diese Form der Sekundärorganisation wurde der Prozess der „Einführung des Unternehmensleitbildes" bereits erwähnt.

2. Es gibt zum anderen Aufgaben, die zwar auch nicht zu den Routineaufgaben der Führungskräfte und Mitarbeiter zählen, die dennoch im Regelfall dauerhaft wahrgenommen werden müssen. Dabei handelt es sich hauptsächlich um Aufgaben des Controllings oder vergleichbare Aufgaben.

Bezogen auf das in Ziffer 1. schon verwendete Beispiel müssen in der Praxis immer wieder unter anderem die folgenden Aufgaben wahrgenommen werden:

- Vielfach haben die Verantwortungsträger bei der praktischen Handhabung des Unternehmensleitbildes Schwierigkeiten: Diese können darauf zurück zu führen sein, dass einzelne Leitsätze des Unternehmensleitbildes nicht präzise genug formuliert worden sind und deshalb neu gefasst werden müssen. Möglicherweise benötigen die Verantwortungsträger zur Überwindung dieser Schwierigkeiten aber nur Hilfestellung, um das Unternehmensleitbild sachgerecht anwenden zu können.

- Vielleicht muss das Unternehmensleitbild, das von der von der Geschäftsführung eingesetzten Arbeitsgruppe erarbeitet worden ist, auch deshalb modifiziert werden, weil sich seit der Einführung des Unternehmensleitbildes die Bedingungen, die $\mathrm{zu}$ seiner damaligen Gestaltung geführt haben, geändert haben.

- In jedem Falle muss aber regelmäßig kontrolliert werden, wie die Verantwortungsträger das Unternehmensleitbild im Sinne der Golden Regel anwenden.

3. Es wurde schon darauf hingewiesen, dass es immer wieder Stakeholder gibt, die aus unterschiedlichen Gründen nicht in der Lage sind, ihre Bedürfnisse mit der für deren Berücksichtigung bei unternehmerischen Entscheidungen hinreichenden Genauigkeit und/oder dem notwendigen Nachdruck geltend zu machen. Diese Stakeholder bedürfen der Unterstützung bei der Wahrnehmung ihrer Interessen.

Die in den Ziffern 2. und 3. skizzierten Aufgaben werden gerne einer Stabsstelle übertragen, die für die Geschäftsführung oder für andere Verant- 
wortungsträger Entscheidungen vorbereitet und/ oder für diese beratend und unterstützend tätig ist. Eine Stabsstelle kann mit einer Person besetzt sein. Sie kann aber auch als multipersonale Instanz organisiert werden.

\section{Patientenfürsprecher und andere Vertreter von Stakeholdern}

In vielen Krankenhäusern gibt es einen Patientenfürsprecher, der die Anliegen der Patienten gegenüber dem Krankenhaus vertritt ${ }^{60}$. Dieser prüft die Anregungen und Beschwerden der Patienten sowie die der Angehörigen, der niedergelassenen Ärzte und diversen Interessengruppen - die Beschwerden resultieren nicht selten aus dem nicht ausreichend Berücksichtigen von Stakeholder-Bedürfnissen bei Entscheidungen der Verantwortungsträger - und sorgt dafür, dass diese bei den einschlägigen Entscheidungen der dafür zuständigen Gremien angemessen berücksichtigt werden.

Darüber hinaus haben viele Krankenhäuser weitere Stellen, meist als Beauftragte bezeichnet, eingerichtet, die entweder die Anliegen einzelner Stakeholder(-gruppen) vertreten oder, bezogen auf bestimmte Aufgabenbereiche, die Interessen diverser Stakeholder im Allgemeinen wahrnehmen. Zu der ersten Cruppe zählen zum Beispiel der Beauftragte für den Elternservice der Charité Universitätsmedizin Berlin, die Personalvertretung, der Ausbildungs-, der Sicherheits- und die Frauenbeauftragte sowie der Schwerbehindertenobmann. Zur zweiten Gruppen gehören z.B. die Datenschutz-, Strahlenschutz- und Brandschutzbeauftragten.

Dem Patientenfürsprecher und den Beauftragten ist gemeinsam, dass sie - wie schon erwähnt die Anliegen, die der einzelne Stakeholder aus unterschiedlichen Gründen nicht hinreichend präzise artikulieren kann und die wegen der häufig sehr heterogen zusammengesetzten Stakeholdergruppe im Detail differieren und deshalb als Einzelmeinung kaum die Chance haben, bei Entscheidungen des Krankenhauses berücksichtigt zu werden, zusammenfassen und in die Sprache

60 So schreibt zum Beispiel $\oint 26$ Abs. 2 Landeskrankenhausgesetz (Berlin) i.d.f. vom 1. März 2001 vor, dass die Bezirksverordnetenversammlung für jedes im jeweiligen Stadt-Bezirk verortete Krankenhaus einen Patientenfürsprecher wählt und einsetzt. der Verantwortungsträger übersetzen. Sie sorgen somit dafür, dass die Anliegen überhaupt wahrgenommen werden. Sie vertreten die selten wirklich mündigen Stakeholder. Sie tragen mit ihrer Arbeit dazu bei, dass es trotz der skizzierten Barrieren zu einer argumentativ, dialogischen Verständigung zwischen dem Verantwortungsträger und den Stakeholdern kommt und dass damit die Anliegen vieler Stakeholder, die sonst unter den Tisch fallen würden, bei den Entscheidungen des Krankenhauses und seiner Verantwortungsträger mit dem für sie angemessenen Gewicht Berücksichtigung finden können.

Patientenfürsprecher und Beauftragte spielen eine Doppelrolle: Sie sind einerseits Interessenvertreter. Zum anderen übernehmen sie eine Beratungsfunktion gegenüber den Verantwortungsträgern und tragen damit dazu bei, dass die Verantwortungsträger ihre Verantwortung gegenüber den verschiedenen Adressaten immer besser - auch ohne die Intervention der Patientenfürsprecher und Beauftragten - wahrnehmen können.

\section{Ethikkomitee}

Die Unterstützung der Geschäftsführung in Angelegenheiten des Managements der sozialen Verantwortung erfolgt sehr häufig durch Ethikkomitees. Dabei werden zwei Arten unterschieden:

1. Die eine Art von Ethikkomitee ist das so genannte Klinische Ethikkomitee ${ }^{6_{1}}$. Dieses berät das therapeutische Team, wenn es Unklarheiten bezüglich der weiteren Behandlung eines Patienten gibt, und erarbeitet Leitlinien für den Umgang mit immer wiederkehrenden klinischen Problemsituationen.

2. Der Wirkungsbereich der anderen Art von Ethikkomitee ist im Sinne der Organisationsethik das gesamte Krankenhaus. Seine Aufgabe ist die Beratung der Geschäftsführung im Umgang mit dem Management der sozialen Verantwortung.

Ausgehend von dem Anliegen der vorliegenden Erörterung ist Gegenstand der weiteren Betrach-

61 Von diesen wiederum zu unterscheiden sind die medizinischen Ethikkommissionen, die zuständig sind für die Beurteilung von Forschungsvorhaben, an denen gesunde Probanden und/oder Patienten beteiligt sind. 
tung nur noch die zweite Art von Ethikkomitee. Dabei werden Antworten zu vier Aspekten erarbeitet (Steinkamp; Gordijn, S. 165ff.):

1. Aufgaben des Ethikkomitees,

2. Arbeitsweise des Ethikkomitees,

3. Stellung des Ethikkomitees in der Krankenhausorganisation und

4. Mitglieder des Ethikkomitees.

\section{Zu 1. Aufgaben des Ethikkomitees}

$\mathrm{Zu}$ den Kernaufgaben des Ethikkomitees zählen (Steinkamp; Gordijn, S. 195):

- Das Ethikkomitee berät die Geschäftsführung in Form von Empfehlungen zur Weiterentwicklung des Unternehmensleitbildes und der Führungsgrundsätze. Die Empfehlungen schließen das Schaffen der personellen und strukturellen Voraussetzungen zur Realisierung des Managements der sozialen Verantwortung ein.

- Diese Beratung erfolgt gefragt, aber auch ungefragt. Die Empfehlungen werden von der Geschäftsführung beschlossen, diffundiert und umgesetzt.

- Zu den Aufgaben gehört das Erarbeiten von Empfehlungen für die inhaltliche Ausgestaltung der Personalentwicklungs-Maßnahmen mit dem Ziel, das Bewusstsein und die Fähigkeit der Führungskräfte und der Mitarbeiter für sozial verantwortliches Handeln zu stärken.

- Das Ethikkomitee ist das Forum innerhalb des Krankenhauses für dialogische Verständigung aller Verantwortungsträger in Angelegenheiten des Managements der sozialen Verantwortung (Steinmann; Löhr 1989, S. 110).

\section{Zu 2. Arbeitsweise des Ethikkomitees}

Von zentraler Bedeutung für die Arbeit des Ethikkomitees ist dessen Unabhängigkeit. Eine Voraussetzung für unbeeinflusstes Arbeiten ist die Trennung zwischen der Argumentationskompetenz des Ethikkomitees und der administrativen Autorität der Geschäftsführung (Steinkamp; Gordijn, S. 174ff.). Das Ethikkomitee - es wurde bereits darauf hingewiesen - erarbeitet Empfehlungen als Grundlage für das Realisieren des Managements der sozialen Verantwortung; die Geschäftsführung setzt diese Empfehlungen in Form von Unternehmensleitlinien und Führungsgrundsätzen um und überwacht deren Einhalten durch die diversen Verantwortungsträger.

Die Arbeitsweise des Ethikkomitees ist grundsätzlich langfristig und institutionell ausgerich- tet. Insofern ist es wichtig, dass seine Mitglieder für eine längere Zeit berufen werden - so z.B. für eine oder auch zwei Legislaturperioden von jeweils drei bis vier Jahren. Die Frequenz der Tagungen des Ethikkomitees hängt von den zu behandelnden Themen und von den jeweiligen Arbeitsfortschritten $\mathrm{ab}$.

Trotz dieser Langfrist-Orientierung darf die gegebenenfalls spontan erforderliche Zusammenarbeit mit den Leistungsbereichen des Krankenhauses nicht vernachlässigt werden. Diese ist nicht nur zu Lösung anstehender Probleme, sondern auch als vertrauensbildende Maßnahme und zur Werbung für die Anwendung der Crundpostulate des Managements der sozialen Verantwortung erforderlich.

In einer Satzung des Ethikkomitees (Steinkamp; Gordijn, S. 174ff.) sind diese Crundsätze etwa wie folgt konkretisiert:

- Die Themen, die von dem Ethikkomitee behandelt werden sollen, werden zu Beginn des Jahres in einen vorläufigen Arbeitsplan mit Prioritäten aufgenommen und mit der Geschäftsführung abgestimmt. Der Arbeitsplan wird durch einen Beschluss der Geschäftsführung zur verbindlichen Arbeitsgrundlage für das Ethikkomitee.

- Das Ethikkomitee berichtet der Ceschäftsführung regelmäßig über die Ergebnisse ihrer Beratungen.

- Das Ethikkomitee hält engen Kontakt zu den Leistungsbereichen des Krankenhauses, sodass die argumentative, dialogische Verständigung zwischen ihm und den Verantwortungsträgern als Voraussetzung für sozial verantwortliches Handeln ermöglicht wird.

- Das Ethikkomitee informiert in Abstimmung mit der Geschäftsführung in regelmäßigen Abständen alle Führungskräfte und Mitarbeiter des Krankenhauses über seine Arbeit.

- Das Unternehmensleitbild und die Führungsgrundsätze werden mittels regelmäßiger Befragungen der Führungskräfte und der Mitarbeiter evaluiert. Dabei geht es vor allem darum herauszufinden, ob das Unternehmensleitbild und die Führungsgrundsätze Handlungsorientierung für die Praxis bieten.

- Darüber hinaus enthält die Satzung Bestimmungen mehr formaler Art, nämlich über den Vorsitz und über die Vorbereitung und Durchführung der Sitzungen. 


\section{Zu 3. Stellung des Ethikkomitees in der Krankenhaus- organisation}

Das Ethikkomitee wird in die Krankenhausorganisation so eingebunden, dass diesem ein möglichst hohes Maß an Freiheit und Unabhängigkeit gewährt ist. Dieses ist die Voraussetzung dafür, dass die Arbeit des Ethikkomitees primär durch die Qualität der Argumente bestimmt wird. Das Ethikkomitee muss so stark mit der Organisation verknüpft werden, dass seine Empfehlungen das notwendige Gewicht haben und deshalb wirkungsvoll umgesetzt werden können. Beide Argumente sprechen dafür, das Ethikkomitee als Stabsstelle der Geschäftsführung direkt zuzuordnen.

Diese enge Anbindung des Ethikkomitees an die oberste Leitungsebene kann allerdings dazu führen, dass das Ethikkomitee von der Geschäftsführung instrumentalisiert wird. Es besteht die Gefahr, dass die Geschäftsführung das Ethikkomitee im Sinne eines bürokratischen Führungsstils missbraucht und sich hinter den Empfehlungen des Ethikkomitees versteckt, indem sie so tut, als sei das Ethikkomitee und nicht sie selbst die entscheidende Instanz. Das Ethikkomitee darf auch nicht im Sinne der Personalführung zweckentfremdet werden und von Führungskräften einen Teil der spezifischen Führungsleistungen übernehmen. Es ist die Aufgabe ausschließlich der Führungskräfte, ihre Mitarbeiter zu sozial verantwortlichem Handeln anzuleiten. Die Trennung zwischen administrativer und argumentativer Kompetenz ist für die Wirksamkeit der Empfehlungen des Ethikkomitees von zentraler Bedeutung.

\section{Zu 4. Mitglieder des Ethikkomitees}

Die personelle Besetzung eines Ethikkomitees ist in der Praxis höchst umstritten. Dabei konzentriert sich die Frage vor allem darauf, ob ein Mitglied der Geschäftsführung Mitglied des Ethikkomitees sein soll oder nicht.

Für die Mitgliedschaft eines Mitgliedes der Geschäftsführung in dem Ethikkomitee spricht, dass dieses durch den Empfehlungsbeschluss des Ethikkomitees verpflichtet ist, die Empfehlung in der Geschäftsführung zu vertreten. Von Vorteil ist auch, dass dadurch die Einbindung in die Organisation gestärkt und die Umsetzung der Empfehlungen des Ethikkomitees gefördert wird.

Die Vertretung der Geschäftsführung im Ethikkomitee ist von Nachteil, weil damit die administrative und argumentative Kompetenz vermengt werden. Die Empfehlungen des Ethikkomitees sind nicht mehr ausschließlich durch die Inhalte des zu lösenden Problems geleitet. Es muss damit gerechnet werden, dass die Umsetzungs-Hürden, die es zweifellos immer gibt, die Beratung des Ethikkomitees beherrschen und das Ergebnis beeinflussen.

Ansonsten prägen zwei Grundprinzipien die personelle Zusammensetzung des Ethikkomitees: Es sollte zum einen dafür gesorgt werden, dass möglichst alle Hierarchieebenen, Berufsgruppen und Leistungsbereiche vertreten sind. Zum anderen empfiehlt es sich, die Handlungsfähigkeit des Ethikkomitees zu sichern, indem die Zahl seiner Mitglieder auf zehn bis maximal fünfzehn Personen beschränkt wird. Wenn in Folge dieser Regelung bei einzelnen Beratungs-Gegenständen der notwendige Sachverstand fehlt, wird dieses Defizit durch das Hinzuziehen von beratenden Mitgliedern, die nicht stimmberechtigt sind, beseitigt.

Die Patienten, deren Angehörige, die Bevölkerung in dem Einzugsgebiet des Krankenhauses sowie die einweisenden Ärzte sind die Stakeholder-Gruppen von zentraler Bedeutung für das Krankenhaus. Es bietet sich deshalb an, Ethikkomitee-Mitglieder nur aus patientennahen Berufsgruppen und Leistungsbereichen zu rekrutieren. Gegebenenfalls werden andere Stakeholder-Cruppen durch den Patientenfürsprecher oder Beauftragte vertreten.

Die Berufung der Ethikkomitee-Mitglieder erfolgt durch die Geschäftsführung. Sofern es ein Ethikkomitee schon gibt und einzelne Mitglieder ausgetauscht werden sollen oder das gesamte Ethikkomitee wegen Ablauf der Legislaturperiode(n) neu besetzt werden soll, hat das gegenwärtige Ethikkomitee ein Vorschlagsrecht. 